\title{
MULTI-FREQUENCY BIOIMPEDANCE AND MYOFASCIAL RELEASE THERAPY: AN EQUINE "ATLASORANGE1" VALIDATION STUDY
}

\author{
Vibeke Sødring Elbrønd ${ }^{1}$, Marta Julia Krasnodebska ${ }^{1}$, Adrian Harrison ${ }^{1}$ \\ ${ }^{I}$ IKVH, Faculty of Health \& Medical Sciences, Gronnegaardsvej 7, 1870
}

Frederiksberg C, Denmark.(vse@sund.ku.dk; zlp973@alumni.ku.dk; adh@sund.ku.dk)

\section{Address Correspondence to:}

Dr Adrian Harrison,

IKVH, Faculty of Health \& Medical Sciences,

Copenhagen University,

Gronnegaardsvej 7,

1870 Frederiksberg C,

Denmark

E-mail: $\underline{\text { adh@sund.ku.dk }}$ 


\begin{abstract}
Background: Measurable sources of muscle tension include viscoelastic tone, physiological contracture, voluntary contraction, and muscle spasm, and if left untreated, they can over a period of time result in clinical pain as a direct result of increased myofascia tension. Typically, physiotherapy is used to stretch affected muscles, thereby reducing motor neuron excitability, and as a consequence give the patient a sensation of correct posture and movement, thereby facilitating normal movement patterns. However, cases of documented therapy effectiveness are rare.
\end{abstract}

Methods: A total of 6 horses with myofascial tension/imbalance issues were selected for this trial. The horses were assessed manually by a qualified Veterinarian and note was taken of regions with high resting myofascial tension as well as movement restrictions. The horses were then measured using a multi-frequency BioImpedance Analysis unit (SFB7) by a technician blinded to the manual assessment. AtlasOrange1, a new form of mechanical physiotherapy massage was used to treat all 6 horses. Treatment was applied directly to two anatomically identifiable myo-fascial regions for a total period of two minutes. Subsequently, the effects of treatment were followed using multi-frequency bioimpedance at an interval of 1, 24 and 48 hours.

Results: Regions of increased myofascial tonus/stiffness were detected manually and noted for all 6 horses. The bioimpedance data was analyzed for centre frequency (fc) and extracellular resistance $(\mathrm{Re})$ and delta values between the right- and left-hand sides of each horse were calculated. Treatment induced a 39\% decrease for Re (NS) and a 58\% decrease for fc $(P=0.003)$ over 48 hours post-treatment. The mfBIA values were subsequently compared with the findings of the manual evaluation revealing a $94.4 \%$ overall agreement.

Conclusion: We suggest that a high level of resting myofascial tension/stiffness is measureable using mf-BIA, and that it can be relieved with massage forms of treatment like AtlasOrange1. (299 of 300 words)

Keywords: myofascia, muscle tension, mf-BIA, mechanical physiothera 


\section{INTRODUCTION}

Muscle tension patterns arise from repeated contractions (Mitsukawa et al. 2009). Thus when certain muscles perform more work than others, imbalances typically occur. Unbalanced riding can also cause some muscles to become overstressed in relation to others, but unilateral tension can also develop as a result of a horse's own compensatory asymmetry. Once established, an imbalance may be emphasized during work, and over time create such noticeable imbalances that they result in physical issues, lameness or pain (Masterson 2011). Studies have found a high prevalence of muscles containing active and latent myofascial trigger points with local mechanical pain sensitivity and referred pain (Sergienko \& Kalichman, 2015), lending support to a myofascial component for many disorders resulting in muscular pain and stiffness (Green et al. 2003).

A recent review of myofascial pain was undertaken by Dommerholt and colleagues (2015) providing a state-of-theart assessment of recent studies addressing this specific topic from Australia, Brazil, Columbia, Denmark, Italy, Iran, Israel, Japan, New Zealand, Qatar, South Korea, Spain, Turkey, the UK and USA. Treatment of myofascial pain involving manual therapy treatment was also recently reviewed by Ajimsha and colleagues (2015). These authors examined the effectiveness of myofascial release, as defined by therapy that involves the application of a low load, long duration stretch to affected myofascial complexes or regions, from studies published in such electronic databases as Medline, Cochrane Library and the Physiotherapy Evidence Database. It was concluded that myofascial release therapy "...shows encouraging effectiveness, emerging as a strategy with a solid evidence base and tremendous potential..." (Ajimsha et al. 2015).

Bioimpedance analysis (BIA) uses the components of impedance (Z): $R$, which is the opposition to the flow of an alternating current through intra-and extracellular ionic solutions, and Xc, which is the delay in the passage of current through the cell membranes and tissue interfaces. Resistance is inversely related to the fluid content, and Xc indicates cell membrane mass, function and interface. BIA therefore enables characterization or classification of relative changes in hydration and cell health/damage in a noninvasive fashion (Nescolarde et al. 2013; Harrison et al. 2015; Bartels et al. 2015).

Apart from $\mathrm{R}$ and $\mathrm{Z}$, mf-BIA can be used to determine the centre frequency (fc), a value that defines the $\mathrm{kHz}$ needed to obtain a maximum Xc value. fc provides important information regarding the relative density of muscle tissue e.g. more or less contracted at rest (Harrison et al. 2015; Bartels et al. 2015). This arises since a contracted, and relatively more dense muscle requires a higher frequency to attain the maximal $\mathrm{Xc}$ value. In addition, values for membrane capacitance $(\mathrm{Mc})$ are achievable, thus an indication of the cell transport activity of a muscle can also be obtained. Furthermore, newer BIA units also allow for a more precise measurement of the extracellular resistance $(\mathrm{Re})$ and the intracellular resistance ( $\mathrm{Ri}$ ), allowing an assessment of localized swelling, dehydration and muscle damage (Harrison et al. 2015; Bartels et al. 2015). Indeed, recently it was shown that a very high correlation $(r=0.89)$ exists between $\mathrm{Ri}$ and $\mathrm{VO}_{2 \max }$ in a cohort of 115 individuals (Stahn et al. 2008). This is an important 
finding as it links cellular oxygen consumption at rest with other parameters obtainable using mf-BIA.

\subsection{Hypotheses}

The hypotheses underpinning this study are; 1) that multi-frequency BIA is capable of detecting contralateral differences in muscle mass, fibre health and level of contraction and metabolic state in horses, 2) that treatment of contralateral muscle differences using an "AtlasOrange1" unit, inducing myofascial muscle release, can be detected using mfBIA, and 3) that treatment using an "AtlasOrange1" unit has both a positive effect on the degree of changes in mf-BIA parameters, as well as their persistence.

\section{MATERIALS AND METHODS 2.2 Subjects}

The equine subjects $(n=6)$ were recruited in southern Sweden (Skaane) with the help of Hans Bohlin and Petra Meller "Atlasbalans AB" (Döbelnsgatan 45, 11352 Stockholm) from a region close to Trelleborg. All horse owners were asked to give their informed consent for the use of mf-BIA. The horses had the following age, weight, breed and activity profiles. Horse 1 was 13 years old, cold blooded, a gelding and used for recreational riding. Horse 2 was 10 years old, cold blooded, a gelding and used for recreational riding. Horse 3 was 9 years old, cold blooded, a stallion and used as a working/drawing horse in the woods and fields. Horse 4 was 10 years old, cold blooded, a gelding and used as a working/drawing horse in the woods and fields (often working in pair with Horse 3). Horse 5 was 11 years old, warm blooded, a gelding and used as a recreational horse. Finally, Horse 6 was 16 years old, a pony, a gelding and used as a recreational horse.

\section{$2.3 m f-B I A$ recordings}

Horses were restrained calmly in a standing position whilst being kept free of all metal surfaces. Precisely determined anatomical areas, e.g. neck, withers, back, hips, abdomen and hind legs were then prepared by shaving. A conductive paste was subsequently applied (Ten20; Weaver and Company, Aurora, Colorado 80011 USA), after which four custom-made pure platinum electrodes $(10 \mathrm{~mm} \times 25 \mathrm{~mm}$; MyoDynamik ApS, DK) were placed on to the prepared sites. An mf-BIA unit (ImpediVET BIS 1, Pinkenba, AU) providing $800 \mu \mathrm{A}$ of current was subsequently attached to the electrodes and recordings carried out.

Measurements were taken over a range of 256 frequencies $(4 \mathrm{kHz}$ to 1000 $\mathrm{kHz}$ ) and repeated six times with a 1 second interval. Using this approach, any slight movement artifacts or changes in the resistance and reactance values due to cable movement, change in the stance, body or electrode movement were minimized. For details concerning the mfBIA recordings see (Harrison et al. 2015; Bartels et al. 2015).

\section{4 mf-BIA data handling}

The mf-BIA recordings were analyzed using the ImpediVET software. Cole-Cole plots were assessed at the time of recording to check for a normal distribution, and both the $\mathrm{R}$ and Xc plots were examined to ensure a precise measurement. Subsequent detailed analyses were then performed for each 
horse using the $50 \mathrm{kHz}$ data and other parameters. These subsequent analyses included the standard parameters of $\mathrm{Z}, \mathrm{R}$ and $\mathrm{Xc}$, as well as the phase angle (PA; $\arctan \mathrm{Xc} / \mathrm{R})$, the $\mathrm{fc}, \mathrm{Re}, \mathrm{Mc}$, and $\mathrm{Ri}$ parameters.

\subsection{Experimental plan}

Each horse was measured 4 times over a period of 48 hours by a person blinded to the site and duration of the treatment. A baseline measurement was taken before treatment, and then a second measurement was made again on the same day, 1 hour after treatment. A third and fourth measurement were subsequently made at 24 hours and 48 hours post treatment, respectively (see TABLE 1).

TABLE 1: An overview of the experimental plan adopted for the mf-BIA recordings of the individual muscle groups on each horse.

\begin{tabular}{|c|c|c|c|}
\hline \multirow{2}{*}{ Baseline } & 1 Hour Post & 24 Hours Post & 48 Hours Post \\
& Treatment & Treatment & Treatment \\
\hline
\end{tabular}

\subsection{Myofascial release therapy}

Myofascial release was undertaken using a multifrequency "AtlasOrange1" device around two different anatomical regions, using the tip of the device. A routine sequence was adopted for each of the regions selected for treatment, and treatment was only applied to the righthand side of each horse. Two identifiable anatomical structures were used as reference points, namely the ala atlantis in the atlanto occipital region, and the tuber coxae. For the ala atlantis, the "AtlasOrange1" was positioned at four sites around this region, three vibration cycles were completed for each site, and the device was angled in order to reach and follow specific myofascial structures. The detailed sequence of vibration cycles for the sites was as follows;

i) caudo dorsal to ala atlantis - in a caudo ventral direction, to contact the myofascia related to $\mathrm{m}$. longissimus capitis;

ii) mid ala atlantis, in a caudo ventral direction, to contact myofascia related to $\mathrm{m}$. splenius;

iii) caudo ventral to ala atlantis, in a caudo ventral direction to contact the myofascia of $\mathrm{m}$. brachiocephalicus, and

iv) cranial to the ala atlantis, in a caudo ventral direction to contact the occipital fascia and m. obl cap. cran.

For the tuber coxae, the "AtlasOrange1" was positioned at five sites around this region, three vibration cycles were completed for each site, and the device was angled in order to reach and follow specific myofascial structures. The detailed sequence of vibration cycles for the sites was as follows;

i) dorso cranial to the tuber coxae, in a cranial direction to contact the thoracolumbar fascia and $\mathrm{m}$. longissimus lumborum; 
ii) midcranial to the tuber coxae, in a medial direction to contact the deeper and hypaxial myofascia and m. iliopsoas

iii) ventrocranial to the tuber coxae in a cranioventral direction to contact the abdominal fasciae and $\mathrm{m}$. obl. int. abd.

iv) mid caudal to the tuber coxae in a ventral direction to contact the fascia lata and $\mathrm{m}$. fascia lata

v) caudo dorsal to the tuber coxae in a caudo ventral direction to contact the fascia and $\mathrm{m}$. gluteus medius.

\subsection{Statistical analyses}

All values are the mean \pm the SD or SEM of muscles or horses as detailed in the figure legends. Differences between means were tested for statistical significance with the use of GraphPad Instat 3 for Mac (Version 3.0b, 2003; GraphPad Inc., La Jolla, CA). All data sets were automatically tested for a Gaussian normal distribution. Differences between means for the individual measurement periods were tested using the TukeyKramer Multiple Comparisons Test, where differences showing a $P$ value $>0.05$ were considered non-significant (NS).

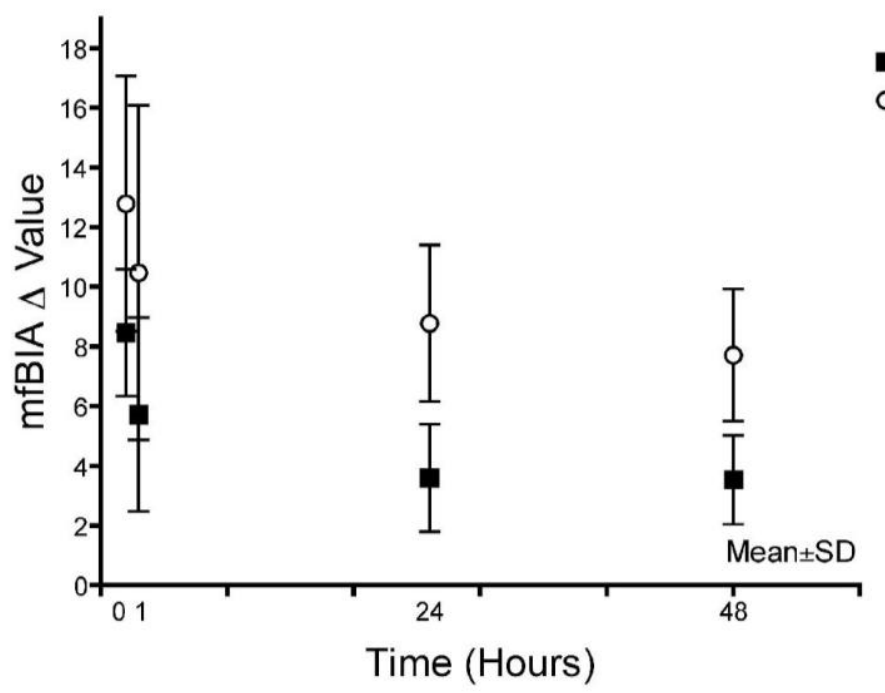

Copyright (C) 2015, Knowledge Enterprises Incorporated. All rights reserved.

\section{RESULTS}

The overall effect of treatment on the mf-BIA delta values for $\mathrm{fc}$ and Re with time post-treatment are presented (see FIG 1). This study observed a rapid decrease in both fc and Re delta values within 1 hour of treatment, compared to baseline values, a change that continued to take effect over the subsequent 24 and 48 hours. This decrease in the difference between fc and Re values on the left compared to the righthand sides of the horses measured in this study, indicates an effect of treatment that improves their muscular symmetry. The delta Re value decreased from 12.8 to 7.7 $\Omega$, a decrease of $39 \%$ (NS) over 48 hours post treatment. In a similar fashion, the delta fc value decreased from 8.4 to 3.5 $\mathrm{kHz}$, a decrease of $58 \%(P=0.003)$ over 48 hours post treatment.

FIG 1: Combined delta values for fc ( $\mathbf{\square}$; $\mathrm{kHz})$ and $\operatorname{Re}(\mathrm{O} ; \Omega) \mathrm{mf}$-BIA parameters between the left and right-hand sides of the measured horses. Overall treatment induced a 39\% decrease for Re (NS) and a $58 \%$ decrease for fc $(P=0.003)$ over 48 hours post-treatment. Values are the mean \pm SD of all the muscles $(n=6)$ and all the horses $(n=6)$ measured.

$$
\begin{aligned}
& \text { fc }(\mathrm{kHz}) \\
& \circ \operatorname{Re}(\Omega)
\end{aligned}
$$


A more detailed investigation of the general response to treatment, revealed a muscle-specific response for the delta fc mf-BIA parameter (see FIG 2). The splenius, internal abdominal oblique, gluteus medius and semitendinosus muscles had a baseline delta value in the range 8.8 to $10.7 \mathrm{kHz}$, whilst the trapezius and longissimus dorsi muscles both showed a lower delta at baseline of 5.5 to $6.1 \mathrm{kHz}$. After 48 hours post treatment, the neck muscle splenius had a delta value that was $42 \%$ of its baseline level, whilst the trapezius muscle remained at its baseline delta value of $6 \mathrm{kHz}$ - unaffected by the treatment. The other muscles, longissimus dorsi, internal abdominal oblique, gluteus medius and semitendinosus all exhibited very similar delta fc values 48 hours post treatment; 2.0 to $3.2 \mathrm{kHz}$.

FIG 2: The mean delta fc $(\mathrm{kHz})$ mf-BIA parameter changes with time for the individual muscle groups measured. Values are the mean \pm SEM of the horses $(\mathrm{n}=6)$ measured.


Likewise, a detailed investigation revealed a muscle-specific response for the of the general response to treatment, Re mf-BIA parameter (see FIG 3). The 
splenius, trapezius, internal abdominal oblique, gluteus medius and semitendinosus muscles had a baseline delta value in the range 10.8 to $18.2 \Omega$, whilst the longissimus dorsi muscle showed a lower delta at baseline of $5.6 \Omega$. After 48 hours post treatment, the same trends were observed. The splenius, trapezius, internal abdominal oblique, gluteus medius and semitendinosus muscles had a delta value in the range 7.3 to $9.3 \Omega$, whilst the longissimus dorsi muscle showed a $37 \%$ lower delta at 48 hours post treatment of $3.5 \Omega$.

FIG 3: The mean delta $\operatorname{Re}(\Omega)$ mf-BIA parameter changes with time for the individual muscle groups measured. Values are the mean \pm SEM of the horses $(n=6)$ measured.

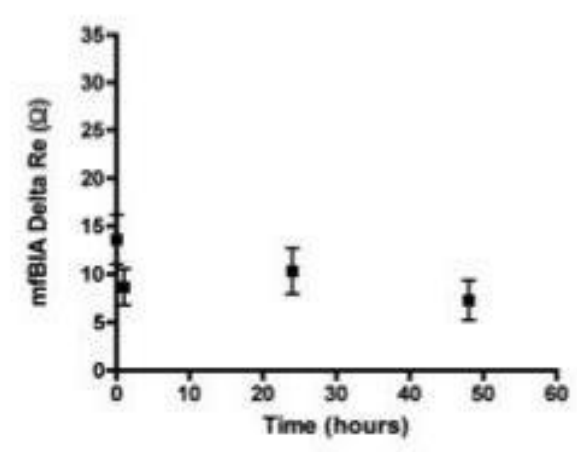

- Splenius
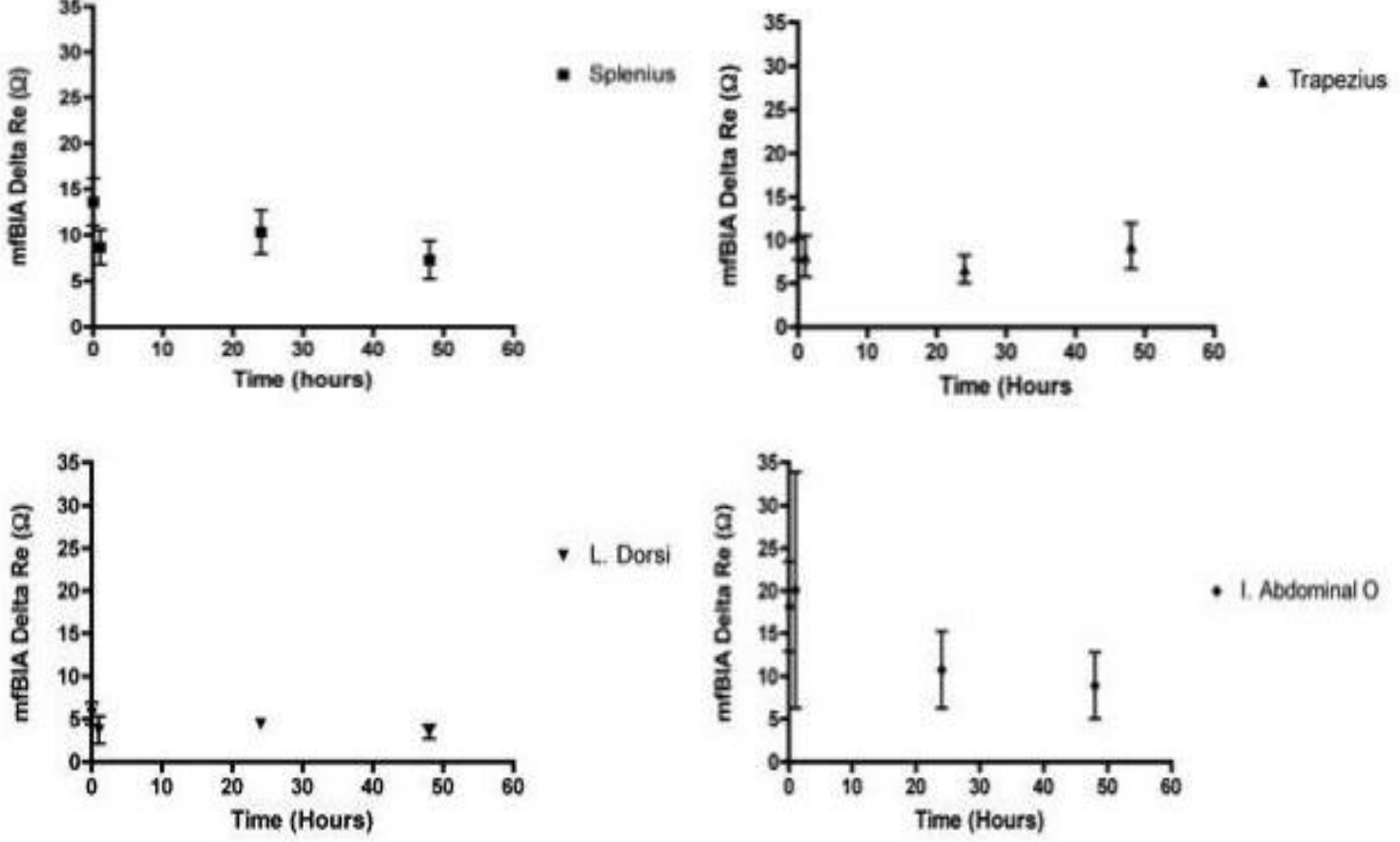

- L. Dorsi
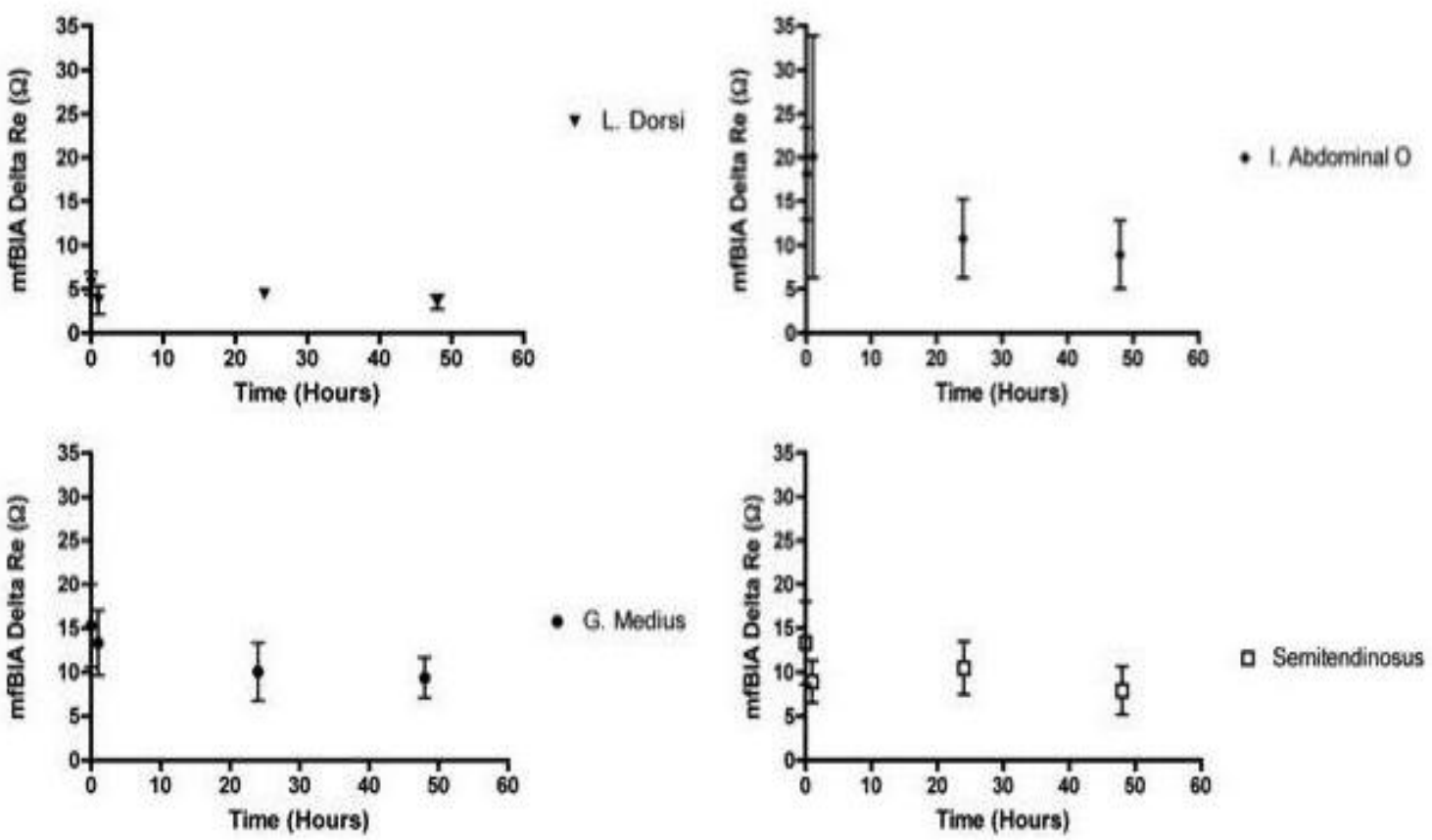

At the level of individual horses, where a clear imbalance was noted pretreatment, "AtlasOrange1" treatment was observed to have effects on associated muscles post-treatment (see FIG 4). Take for example the fourth horse measured, which was a working horse that presented

with a pre-treatment imbalance in the fc parameter of $22.7 \mathrm{kHz}$ for the internal abdominal oblique muscle. One hour after treatment using the "AtlasOrange1" unit and the imbalance in the particular muscle had fallen to just $8.7 \mathrm{kHz}$ and by 24 hours it had fallen again to $4.2 \mathrm{kHz}$ and by 48 
hours the imbalance was all but gone $(0.1$ $\mathrm{kHz}$ ). This release of tension (left versus right) at the level of the internal abdominal oblique muscle initially induced an imbalance in the trapezius muscle and to some extent also in the gluteus medius muscle (1 hour post-treatment), effects that were transitory and had disappeared by 24 hours post-treatment.

FIG 4: In panel A: The individual delta fc $(\mathrm{kHz})$ mf-BIA values for each muscle (left versus right) pre- as well as 1, 24 and 48 hours post-treatment for Horse No. 4 are presented. Note the muscles with the highest level of imbalance (left versus right) pre-treatment, induce an imbalance in associated muscles after treatment something that slowly dissipates over the 48 hour period post-treatment. Values represent an imbalance between the left and right sides of this horse. In panel B: A Picture of Horse No. 4, shows how the trunk was rotated axially towards the right at pre-treatment, and also provides an illustration of regions of tonus/contraction or stiff fascia (blue circles) as assessed by independent assessment of muscular tonus made manually by a qualified Veterinarian prior to treatment. Regions of tonus/contraction were found to closely correlate with high delta fc $(\mathrm{kHz})$ mf-BIA values.

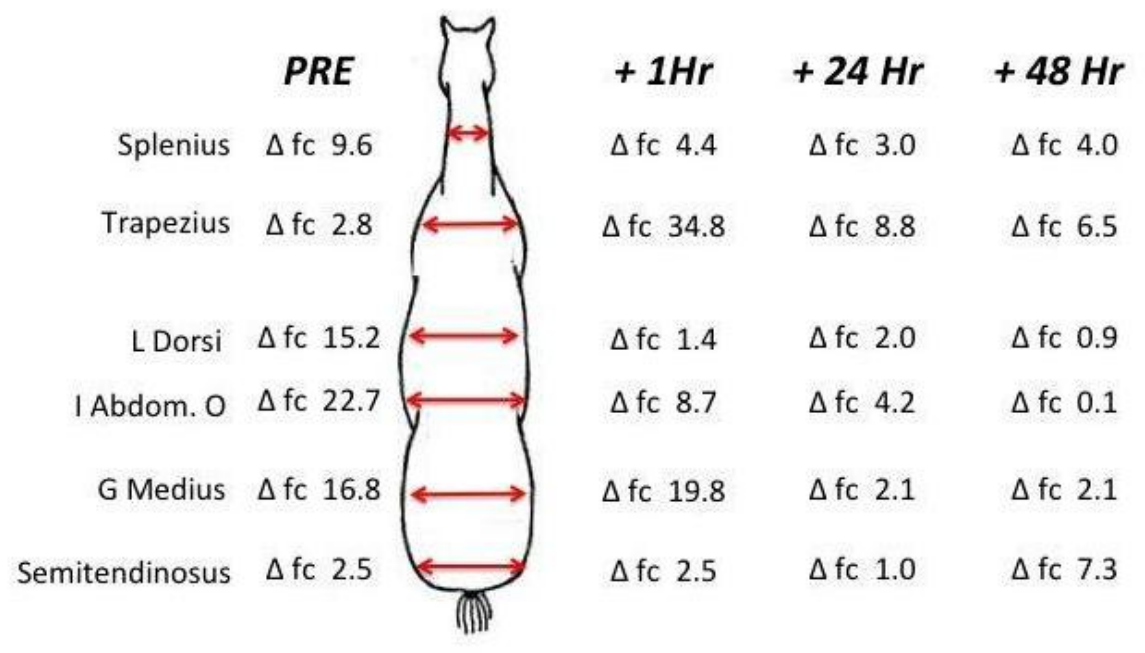

B
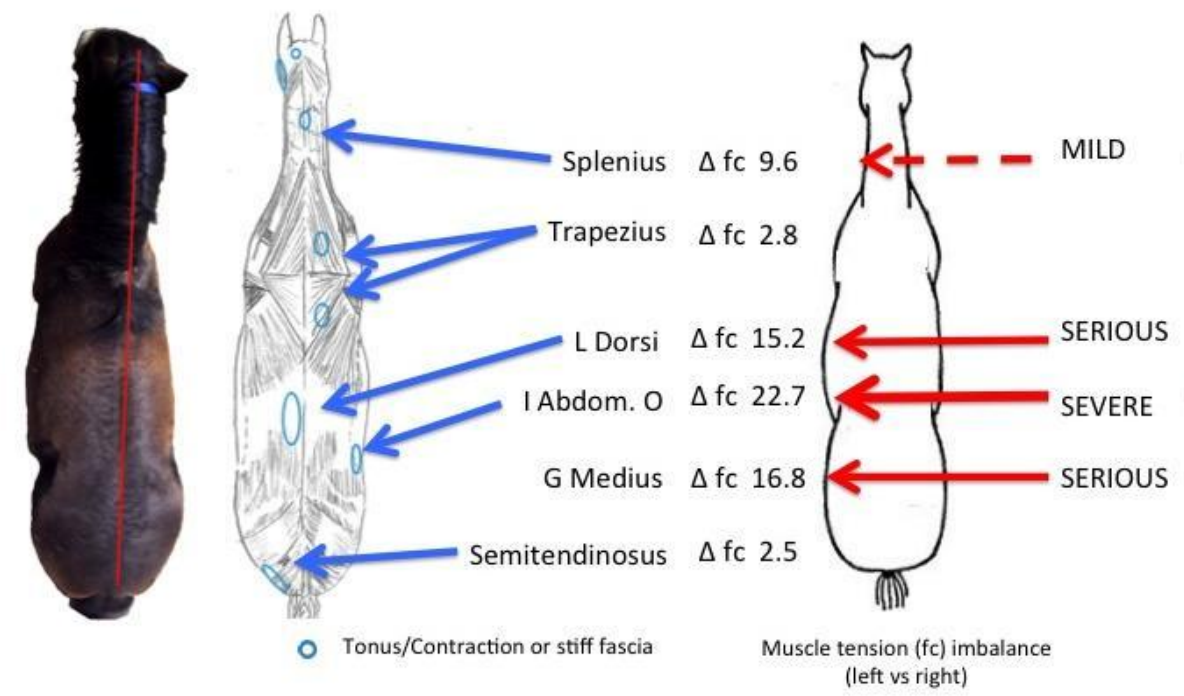
Similar effects to those found for Horse number 4 , were observed in $50 \%$ of the horses measured, typically in connection with release of a region with a relatively high imbalance.

measured and treated using "AtlasOrange1" were found to have an imbalance in either the fc or the $\mathrm{Re}$ parameter in four or more of the muscle regions measured in this study. The most common muscle regions in terms of imbalance were found to be the trapezius, internal abdominal oblique and gluteus medius muscle groups.

An independent assessment of muscular tonus/fascia stiffness was made manually by a qualified Veterinarian. This assessment included both increased regional muscle and fascia related tonus, which was subsequently analyzed with regard to the delta fc mf-BIA parameter. The results revealed an overall comparison of $94.4 \%$ for mf-BIA fc indications of muscular contraction and regional muscular tonus/fascia stiffness when compared with a manual assessment of increased regional muscular or fascia tonus (see FIG 4B). Moreover, the comparisons were found for all 6 of the horses examined in this study. The manual assessment of muscular tonus/fascia stiffness identified an additional 2 regions in one horse and 1 additional region in 4 horses, besides those detected using mfBIA.

With regard to Horse 4 (see FIG 4B) there is a very clear sign of axial rotation of the trunk towards the right hand side (see FIG 4B - photo). It is also worth mentioning that this horse had difficulty with head, neck and trunk extension, as well as thorax and lumbar flexion. The lumbar rotation from the right-hind to left- front was reduced/stiff, and the diagonal movement from the left-hind towards the right shoulder was also reduced/stiff, likewise lateral flexion towards the left for the entire trunk region. It is interesting, but not surprising to note that this horse, which always worked together with Horse 3, preferred to be positioned on the righthand side of the pair.

\section{DISCUSSION}

To the best of the authors knowledge, this is the first study to apply the non-invasive technique of mf-BIA to validate the effectiveness of myofascial release therapy. It not only shows how certain key mf-BIA parameters almost immediately respond to a relatively short period of treatment, but that the effects of myofascial release can have prolonged benefits for those subjects treated.

The technique of mf-BIA has been used for the past three years by one of the co-authors $(\mathrm{AH})$ in the measurement of more than 150 horses, comprising both healthy and trained controls, as well as injured animals. It has been proven to be a repeatable and accurate technique, both in the human and in the veterinary clinics (Nescolarde et al. 2013; Harrison et al. 2015; Bartels et al. 2015).

This study has shown an effect of myofascial release therapy on a number of muscle groups in six horses. In a recent study involving 186 patients undertaken by Licciardone \& Aryal (2014), the effects of osteopathic manual treatment on chronic lower back pain were assessed. This study revealed a large effect size of short-term efficacy of osteopathic manual treatment on chronic lower back pain, an effect that was driven by stable responders who did not relapse (Licciardone \& Aryal, 2014). 
These authors found that osteopathic manual treatment gave a clinical response after three scheduled treatment sessions delivered over a period of four weeks, however, no quantifiable assessment was made, instead treatment response was evaluated by means of a VAS pain score completed by the patients. In the present study, mf-BIA and the centre frequency (fc) parameter were used to quantify resting muscle tension or tonus, to determine any left versus right imbalance and to follow the effects of treatment. The present data show not only regions of imbalance in the muscle groups of the horses examined, but also the effects of myofascial release treatment using the "AtlasOrange1" unit. Moreover, these effects of treatment were very often of a rapid nature, occurring within an hour of treatment, 24 hours or 48 hours of treatment.

In another patient trial, involving 374 individuals (79\% male; mean age 43 years; mean symptom duration 5 years) the effects of pelvic floor physical therapy were assessed in terms of the medication used to treat chronic pelvic pain syndrome (Anderson et al. 2015). Anderson and colleagues (2015) were able to show a significant voluntary cessation in the use of oral medications associated with the use of an internal trigger point wand aimed at alleviating internal pelvic floor myofascial pain. Indeed, the use of an internal trigger point wand resulted in an $86 \%$ reduction in the number of patients suffering from trigger point sensitivity, something that was achieved within a 6 month time frame. One fault with the Anderson and colleagues (2015) trial, however, is that medication histories were self-reported, and this opens their data up for recall bias issues. In the present study, the use of mf-
BIA parameter changes alone (delta fc) were used to quantify the effects and response of mechanical myofascial release treatment, and these changes were additionally monitored by means of a pictorial record of a change in posture and anatomical alignment. In this way, the present study and its findings are not open to patient derived bias.

The effects of "AtlasOrange1" treatment in the present study were found to be relatively rapid if not to say immediate. In a trial of the effects of suboccipital muscle inhibition and selfmyofascial release on hamstring shortening in 50 human subjects, Cho and colleagues (2015) also reported immediate effects. The subocciptal muscle inhibition technique induces relaxation of the fascia by applying soft pressure by a therapist, to the subocciptal area of a patient while they are lying down. In contrast, self-myofascial release involving the use of a foam roller applied in this case to the hamstring muscles means that treatment can be undertaken almost anywhere by the patients themselves. It is known that shortening of a muscle, in this case the hamstrings, can have a negative effect on posture (Park et al. 2012). Indeed, an increase in stiffness of the hamstring muscles has been associated with lower back pain (Gajdosik et al. 1992).

In the study performed by Cho and colleagues (2015), it was found that treatment had an immediate effect (5 minutes post treatment) in terms of hamstring relaxation and flexibility as determined by the finger-to-floor-distance test (a subject stands and bends down without bending their knees, and the distance between their fingertips and the floor is measured). However, of greater interest was their finding that the 
suboccipital muscle inhibition treatment was more effective that the self-myofascial release treatment. The authors explain this difference in response in part to a difference in the density of muscle spindles in the muscles treated (subocciptal muscle $>$ hamstrings) and state that the number of muscle spindles a muscle has is closely related to its role in the regulation of posture and degree of tension (Peck et al. 1984). However, the authors also write that "...what is needed for effective relaxation of the fascia is proper pressure and soft extension on the area where fascia limitation is felt..." raising the point of the mode of action of myofascial release (Cho et al. 2015). It is interesting in the present study, where the effect of a mechanical form of myofascial release therapy has also resulted in a very rapid, if not immediate response, that manual versus mechanical treatment is not important - rather perhaps, it is the effectiveness of the relaxation and soft extension of the affected fascia that is critical for successful treatment as stated by Cho and colleagues (2015)?

\section{PERSPECTIVES}

The presence of myofascial trigger points in muscles associated with pain and stiffness raises the possibility that the myofascial component may well be the primary or secondary source of muscle pain (Sergienko \& Kalichman, 2015). Clearly, additional studies are now needed to address not only the contributing factors causing myofascial trigger point development in affected muscles, but also the mode and duration of treatment posed by for example myofascial release therapy. The present results, however, now provide evidence that not only indicates that myofascial release therapy induces quantifiable changes in skeletal muscles, but that such changes are also relatively immediate and long lasting.

\section{ACKNOWLEDGEMENTS}

The authors wish to thank the owners for permitting these measurements on their horses. This study was funded by "Atlasbalans AB" Döbelnsgatan 45, 11352 Stockholm, Sweden (hans.bohlin@atlasbalans.se;

petra.meller@atlasbalans.se) although all measurements were performed by an operator blinded to the site of treatment on each horse. mf-BIA recordings were undertaken by MyoDynamik ApS without any conflict of interest. Hans Bohlin is the owner of "Atlasbalans AB" whilst Petra Meller is an expert in myofascial release therapy in both humans and horses, and holds courses in the use of "AtlasOrange1". 


\section{REFERENCES}

Ajimsha, M.S., Al-Mudahka, N.R., AlMadzhar, J.A. 2015. Effectiveness of myofascial release: Systematic review of randomized controlled trials. Journal of Bodywork \& Movement Therapies 19: 102-112.

Anderson, R.U., Harvey, R.H., Wise, D., Smith, J.N., Nathanson, B.H., Sawyer, T. 2015. Chronic pelvic pain syndrome: Reduction of medication use after pelvic floor physical therapy with an internal myofascial trigger point wand. Appl. Psychophysiol. Biofeedback DOI 10.1007/s10484-015-9273-1.

Bartels, E.M., Sørensen, E.R., Harrison, A.P. Multi-frequency bioimpedance in human muscle assessment. Physiological Reports. In Press.

Cho, S-H., Kim, S-H., Park, D-J. 2015. The comparison of the immediate effects of application of the suboccipital muscle inhibition and self-myofascial release techniques in the suboccipital region on short hamstring. J. Phys. Ther. Sci. 27: 195-197.

Dommerholt, J., Grieve, R., Layton, M., Hooks, T. 2015. An evidence-informed review of the current myofascial pain literature - January 2015. Journal of Bodywork \& Movement Therapies 19: 126-137.

Gajdosik, R.L., Hatcher, C.K., Whitsell, S. 1992. Influence of short hamstring muscles on the pelvis and lumbar spine in standing and during the toe-touch test. Clin. Biomech. 7: 38-42.
Green, S., Buchbinder, R., Hetrick, S. 2003. Physiotherapy interventions for shoulder pain. Cochrane Database Syst. Rev. 2, CD004258.

Harrison, A.P., Elbønd, V.S., Riis-Olesen, K., Bartels, E.M. 2015. Multi-frequency Bioimpedance in equine muscle assessment. Physiol. Meas. 36:453-464.

Licciardone, J.C., Aryal, S. 2014. Clinical response and relapse in patients with chronic low back pain following osteopathic manual treatment: Results from the OSTEOPATHIC Trial. Manual Therapy 19: 541-548.

Masterson, J. 2011. Beyond Horse Massage: A breakthrough interactive method for alleviating soreness, strain and tension. Trafalgar Square Books, ISBN 9781570764721.

Mitsukawa, N., Sugiaski, N., Kanehisa, H., Fukunaga, T., Kawakami, Y. 2009. Fatigue-related changes in fascicle-tendon geometry over repeated contractions: Difference between synergist muscles. Muscle \& Nerve, 40: 395-401.

Nescolarde, L., Yanguas, J., Lukaski, H., Alomar, X., Rosell-Ferrer, J., Rodas, G. 2013. Localized bioimpedance to assess muscle injury. Physiol. Meas. 34: 237-245.

Park, K.N., Yi, C.H., Jeon, H.S., Lee, W.H., Ha, S.M., Kim, S.J., Kwon, O.Y. 2012. Effects of lumbopelvic neutralization on the electromyographic activity, lumbopelvic and knee motion during seated knee extension in subjects with hamstring shortness. J. Phys. Ther. Sci. 24: 17-22. 
Peck, D., Buxton, D.F., Nitz, A. 1984. A comparison of spindle concentrations in large and small muscles acting in parallel combinations. J. Morphol. 180: 243-252.

Sergienko, S., Kalichman, L. 2015. Myofascial origin of shoulder pain: A literature review. Journal of Bodywork \& Movement Therapies 19: 91-101.

Stahn, A., Strobel, G., Terblanche, E. 2008. $\mathrm{VO}_{2 \max }$ prediction from multifrequency bioelectrical impedance analysis. Physiol. Meas. 29: 193-203. 